\title{
Negative clinical trials in sarcoidosis: failed therapies or flawed study design?
}

\author{
David R. Moller \\ Affiliation: Johns Hopkins University School of Medicine, Baltimore, MD, USA.
}

Correspondence: David R. Moller, Johns Hopkins University School of Medicine, 5501 Hopkins Bayview Circle, Baltimore, MD 21042, USA. E-mail: dmollerajhmi.edu

@ERSpublications

Negative results of clinical trials in sarcoidosis provide insights that can be used to rethink study design http://ow.ly/AWuWw

In this issue of the European Respiratory Journal, JUDSON et al. [1] report the results of a large clinical trial involving two new potential therapies in sarcoidosis, a subcutaneous anti-tumour necrosis factor (TNF) agent, golimumab, and a novel anti-p40 (a component of both interleukin (IL)-12 and IL-23) agent, ustekinumab. The rationale for the use of anti-TNF agents in sarcoidosis is compelling, as TNF plays a central role in granuloma formation [2]. The use of anti-p40 (anti-IL-12/IL-23) also has a solid rationale based on the role of IL-12 in promoting T-helper (Th) type 1 immune responses and its upregulation in sarcoidosis. The role of the IL-23/Th17 pathways in sarcoidosis is less well established but the benefits of blocking IL-23 together with IL-12 is conceptually appealing in sarcoidosis [3].

Given the clear rationale for testing these therapies in sarcoidosis, it is disappointing that neither therapy showed clinical benefit assessed by either primary or secondary end-points. These negative results follow a large trial of infliximab by many of these same investigators. In a randomised, placebo-controlled trial of infliximab in pulmonary sarcoidosis by BAUGHMAN et al. [4], there was a statistically significant $2.5 \%$ increase in forced vital capacity (FVC) \% predicted with infliximab versus placebo and no significant differences for any of the major secondary end-points at 6 months. Although the authors suggest this was a robust response, healthcare payers (both corporations and governments) concluded that infliximab demonstrated only minimal improvement for patients with pulmonary sarcoidosis and cite this research as a reason to deny coverage of this therapy. Several studies find infliximab beneficial for progressive nonpulmonary manifestations of sarcoidosis $[5,6]$, but in aggregate, these studies have not led to regulatory approval for infliximab in sarcoidosis in either Europe or the USA.

The negative results of the current study, together with the marginal benefit of infliximab shown in pulmonary sarcoidosis, begs the question of whether these therapies are truly ineffective or they failed because of flaws in the study design. The challenge in designing clinical trials in sarcoidosis is the limited database of randomised, placebo-controlled studies from which to draw lessons. Nonetheless, clinical trial design must take into account disease-specific pathobiology. In this context, a review of the clinical features of sarcoidosis and response to corticosteroid therapy is indicated.

Master clinicians working in an era during which therapy of sarcoidosis was restricted to corticosteroids (and sometimes antimalarials) taught that the following observations are relevant to most $(>90 \%)$ patients with chronic pulmonary sarcoidosis [7]. 1) Corticosteroid therapy is highly effective for both short- and long-term control if given in a sufficient dose, but is associated with significant adverse effects. 2) The minimum effective corticosteroid dose is precisely defined, and differs from patient to patient and for different organs. For chronic pulmonary sarcoidosis, the range is typically between 5 and $15 \mathrm{mg}$ per day (most often 10-15 mg). For cardiac sarcoidosis, the range is typically between 10 and $20 \mathrm{mg}$ per day (most

Received: Aug 262014 | Accepted: Aug 272014

Conflict of interest: Disclosures can be found alongside the online version of this article at erj.ersjournals.com

Copyright (CERS 2014 
often 15-20 mg). Even 1-2-mg dosing can make the difference between effective long-term control and progressive inflammation with organ dysfunction. 3) The minimum effective corticosteroid dose does not vary with time. 4) Pre-existing fibrosis is irreversible, and determines baseline function and symptoms while on an effective maintenance dose (persistent symptoms may not mean inadequate suppression). 5) Recurrent (or relapsing) granulomatous inflammation in chronic pulmonary sarcoidosis is due to tapering below an effective maintenance corticosteroid dose. 6) Recurrent sarcoidosis inflammation typically takes weeks to several months to become clinically evident given the slow progression of granulomatous inflammation (i.e. there is a lag period between tapering below an effective corticosteroid dose and clinically evident relapsing pulmonary sarcoidosis). More recent experience suggests that 7) immunomodulatory, non-corticosteroid sparing therapies are effective in a subset of patients, enabling a reduction in the minimum effective corticosteroid dose, but often, a small corticosteroid dose is needed for stable, long-term effectiveness [8]. These sarcoidosis features are not hypotheses; rather, they reflect clinical observations that characterise the unique pathobiological responses of sarcoidosis.

Viewing the current study and prior infliximab trial within the disease-specific characteristics of sarcoidosis provides a context to examine whether the study design could have influenced the negative outcomes. In these studies, the primary intervention was added after the subject was documented to be stable on maintenance corticosteroid therapy (prednisone $10-25 \mathrm{mg}$ per day) for $\geqslant 1$ month, which was continued throughout phase I. Thus, in order for a therapy to meet its primary end-point (change in FVC \% predicted), either the therapy must provide an additional functional benefit on top of the stable corticosteroid regimen, or the placebo arm subjects needed to deteriorate from their baseline while subjects with therapeutic intervention remained stable. Both requirements are inherently flawed, which is now illustrated by the negative study results.

First, there is no clinical or scientific basis for the concept that add-on therapy will improve lung function over baseline in chronic sarcoidosis subjects who already have their granulomatous pulmonary inflammation effectively suppressed by a corticosteroid-containing regimen. Consistent with this observation, a post hoc analysis of the infliximab study is notable in finding that subjects on prednisone $\geqslant 15 \mathrm{mg}$ per day saw no benefit from infliximab [9]. This result is explained by the fact that prednisone $\geqslant 15 \mathrm{mg}$ per day is effective maintenance therapy for most patients with chronic pulmonary sarcoidosis. Patients with chronic pulmonary sarcoidosis on effective, stable maintenance therapy may have persistent respiratory symptoms due to pre-existing irreversible fibrosis and lung impairment, which will not be impacted by anti-inflammatory therapies. Although some measures of inflammation may remain elevated on functionally effective corticosteroid regimens, there is no basis for an add-on functional benefit once effective suppression of granuloma formation in the lung is achieved.

Second, there is no basis for the expectation that pulmonary sarcoidosis subjects on stable corticosteroid maintenance therapy will deteriorate over the time course of these clinical trials. Both the current trial and infliximab study demonstrate this fact by the lack of significant change in FVC in the placebo arms in either study. Why then did the infliximab study show a small but statistically significant improvement in FVC with infliximab? A post hoc analysis showed that most benefit was seen in those subjects with most severe disease [4]. A simple explanation is that this smaller subset of subjects was not truly stable on maintenance therapy and thus benefitted from the added anti-TNF effects over the longer 6-month intervention timeframe compared with the 1-month run-in period.

Perhaps both assumptions derive from a muddled notion that pulmonary sarcoidosis can undergo acute exacerbations with a "flare" from a baseline state of activity or suppression analogous to idiopathic pulmonary fibrosis [10]. New granuloma formation in sarcoidosis takes weeks to develop, as seen in the Kveim reaction [11]. Relapses in chronic pulmonary sarcoidosis are mostly caused by tapering below or off an effective anti-inflammatory regimen with clinically progressive granulomatous lung inflammation occurring after a time lag of weeks to months; acute bronchospasm can occur but results from the underlying progressive sarcoidosis inflammation [12-14]. Consistent with this observation, relapsing pulmonary sarcoidosis responds to low-dose corticosteroids typically seen in maintenance treatment responses [15]. However, relapsing pulmonary sarcoidosis is neither acute nor a true exacerbation (implying a sudden increase in granulomatous inflammation or sudden lack of response to maintenance therapy). Acute respiratory exacerbations in sarcoidosis are largely due to non-sarcoidosis related issues such as respiratory infections or complications of nonpulmonary sarcoidosis manifestations.

The benefit of infliximab in some subjects with nonpulmonary manifestations can be explained by the observation that these manifestations may not respond as readily to corticosteroid doses that are effective for pulmonary sarcoidosis. In the infliximab study, subjects enrolled based on stable maintenance corticosteroid therapy for pulmonary sarcoidosis could have active, progressive nonpulmonary manifestations despite their therapy. Since active, nonpulmonary, organ-specific granulomatous 
inflammation was incompletely suppressed, a test of efficacy could be accomplished with infliximab demonstrating effectiveness using a novel organ severity tool [5].

The authors correctly suggest that corticosteroids may have masked the effect of the anti-TNF golimumab, given the lack of effect in phase I with maintenance corticosteroid therapy and the trend toward a steroidsparing effect in phase II. The more significant point is that stable maintenance corticosteroid regimens will mask the effect of any anti-inflammatory/immunomodulatory therapy on chronic pulmonary sarcoidosis, whether anti-TNF, anti-IL-12/IL-23, old, new or yet-to-come therapies.

Other study designs have used a steroid-sparing (taper) phase at the beginning of the intervention phase [16]. This design has the advantage of mimicking how these steroid-sparing therapies are used in clinical practice. The authors of the current study tacked on an exploratory steroid taper in phase II after the primary end-point and important lessons can be gleaned from the results. Hints of effectiveness were seen for golimumab in skin sarcoidosis and as a steroid-sparing therapy in pulmonary sarcoidosis, but statistically significant end-points were not met. As the authors suggest, a test of effectiveness was likely hampered by the inadequate timeframe for assessing steroid-sparing benefit in pulmonary sarcoidosis after corticosteroid taper. The inadequate timeframe may have been exacerbated by the fact that some of these subjects were probably still on an effective corticosteroid dose (e.g. 5-12.5 mg per day) during this 3-month phase, suggested by the number of patients on placebo who were able to taper prednisone without any subsequent deterioration in their pulmonary function.

The optimal steroid-sparing trial design in sarcoidosis is unclear. Based on sarcoidosis pathobiology, the study design should use a sufficient run-in period (or history) with an effective corticosteroid dose (e.g. prednisone 15 or $20 \mathrm{mg}$ per day for $\geqslant 2$ months) to assure maximum and stable baseline function, a mandated steroid taper at the beginning of the intervention to a prescribed low corticosteroid dose (e.g. prednisone $5 \mathrm{mg}$ per day) and a minimum 4-6-month time frame after steroid taper that is sufficient to predict most subjects on placebo will deteriorate from their baseline based on prior history. Effective steroid-sparing therapy would demonstrate stable pulmonary end-points.

Other mitigating features of the study design involve the primary end-point [17]. The use of percentage change in predicted FVC as a single end-point does not capture the heterogeneous manifestations of pulmonary sarcoidosis. In sarcoidosis, obstructive impairment is frequent and a sizable subset of pulmonary sarcoidosis patients has a dominant reduction in diffusing capacity compared with spirometric measures [18]. FVC is a poor measure of the dominant pathophysiology in these latter patients, resulting in reduced power for the trial unless excluding these latter groups of subjects. The best way to capture clinically significant changes in pulmonary function in these different subgroups of patients remains uncertain. Changes in chest radiographs have been proposed along with FVC as a primary end-point [19] but lack the sensitivity of pulmonary function testing. The study explored several biomarkers as surrogate markers of a therapeutic response. Although of considerable interest from a mechanistic viewpoint, the uncertain correlation of these biomarkers with functional outcomes makes it unlikely these or other biomarkers are ready to replace functional end-points.

The stakes are high. Additional therapies are greatly needed in sarcoidosis that are effective, have a reasonable safety profile and can reduce or replace the need for long-term corticosteroid therapy in those with chronic sarcoidosis who experience the inevitable development of corticosteroid toxicities [20]. If the current study design is widely adopted, the sarcoidosis community faces the prospect of more negative studies regardless of therapeutic promise. Fortunately, the results of these negative studies provide important insights that can be used as a springboard to rethink study design before other promising therapies undergo expensive and time-consuming clinical trials.

\section{References}

1 Judson MA, Baughman RP, Costabel U, et al. Safety and efficacy of ustekinumab or golimumab in patients with chronic sarcoidosis. Eur Respir J 2014; 44: 1296-1307.

2 Zissel G, Prasse A, Muller-Quernheim J. Immunologic response of sarcoidosis. Semin Respir Crit Care Med 2010; 31: 390-403.

Chen ES, Moller DR. Sarcoidosis-scientific progress and clinical challenges. Nat Rev Rheumatol 2011; 7: 457-467. Baughman RP, Drent M, Kavuru M, et al. Infliximab therapy in patients with chronic sarcoidosis and pulmonary involvement. Am J Respir Crit Care Med 2006; 174: 795-802.

5 Judson MA, Baughman RP, Costabel U, et al. Efficacy of infliximab in extrapulmonary sarcoidosis: results from a randomised trial. Eur Respir J 2008; 31: 1189-1196.

6 Stagaki E, Mountford WK, Lackland DT, et al. The treatment of lupus pernio: results of 116 treatment courses in 54 patients. Chest 2009; 135: 468-476.

7 Johns CJ, Michele TM. The clinical management of sarcoidosis. A 50-year experience at the Johns Hopkins Hospital. Medicine (Baltimore) 1999; 78: 65-111. 
Vorselaars AD, Wuyts WA, Vorselaars VM, et al. Methotrexate vs azathioprine in second-line therapy of sarcoidosis. Chest 2013; 144: 805-812.

9 Judson MA, Baughman RP, Costabel U, et al. The potential additional benefit of infliximab in patients with chronic pulmonary sarcoidosis already receiving corticosteroids: a retrospective analysis from a randomized clinical trial. Respir Med 2014; 108: 189-194.

10 Panselinas E, Judson MA. Acute pulmonary exacerbations of sarcoidosis. Chest 2012; 142: 827-836.

11 Munro CS, Mitchell DN. The Kveim response: still useful, still a puzzle. Thorax 1987; 42: 321-331.

12 Vorselaars AD, Verwoerd A, van Moorsel $\mathrm{CH}$, et al. Prediction of relapse after discontinuation of infliximab therapy in severe sarcoidosis. Eur Respir J 2014; 43: 602-609.

13 Panselinas E, Rodgers JK, Judson MA. Clinical outcomes in sarcoidosis after cessation of infliximab treatment. Respirology 2009; 14: 522-528.

14 Gottlieb JE, Israel HL, Steiner RM, et al. Outcome in sarcoidosis. The relationship of relapse to corticosteroid therapy. Chest 1997; 111: 623-631.

15 McKinzie BP, Bullington WM, Mazur JE, et al. Efficacy of short-course, low-dose corticosteroid therapy for acute pulmonary sarcoidosis exacerbations. Am J Med Sci 2010; 339: 1-4.

16 Park MK, Fontana Jr, Babaali H, et al. Steroid-sparing effects of pentoxifylline in pulmonary sarcoidosis. Sarcoidosis Vasc Diffuse Lung Dis 2009; 26: 121-131.

17 Baughman RP, Drent M, Culver DA, et al. Endpoints for clinical trials of sarcoidosis. Sarcoidosis Vasc Diffuse Lung Dis 2012; 29: 90-98.

18 Valeyre D, Prasse A, Nunes H, et al. Sarcoidosis. Lancet 2014; 383: 1155-1167.

19 Baughman RP, Shipley R, Desai S, et al. Changes in chest roentgenogram of sarcoidosis patients during a clinical trial of infliximab therapy: comparison of different methods of evaluation. Chest 2009; 136: 526-535.

20 Baughman RP, Nunes H, Sweiss NJ, et al. Established and experimental medical therapy of pulmonary sarcoidosis. Eur Respir J 2013; 41: 1424-1438. 